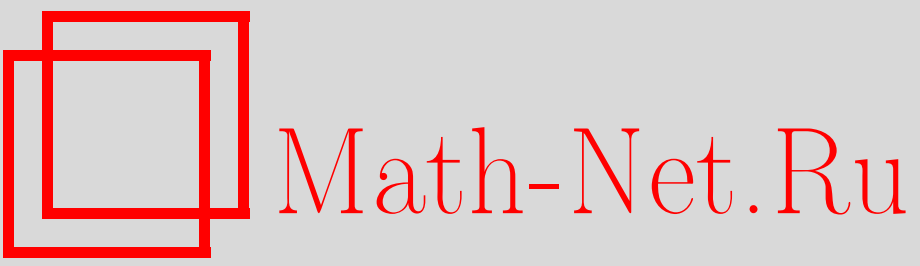

А. В. Щепетилов, Квантово-механическая задача двух тел с центральным взаимодействием на односвязных поверхностях постоянной кривизны, ТМФ, 1999, том 118, номер 2, 248-263

DOI: https://doi.org/10.4213/tmf697

Использование Общероссийского математического портала Math-Net.Ru подразумевает, что вы прочитали и согласны с пользовательским соглашением

http: //www . mathnet.ru/rus/agreement

Параметры загрузки:

IP : 54.80 .97 .219

26 апреля 2023 г., 11:49:05 
ТЕОРЕТИЧЕСКАЯ

И МАТЕМАТИЧЕСКАЯ

ФИЗИКА

Том 118, № 2

февраль, 1999

(C) $\mathbf{1 9 9 9}$ г.

А.В. Шепетилов*

КВАНТОВО-МЕХАНИЧЕСКАЯ

\section{ЗАДАЧА ДВУХ ТЕЛ С ЦЕНТРАЛЬНЫМ \\ ВЗАИМОДЕЙСТВИЕМ НА ОДНОСВЯЗНЫХ ПОВЕРХНОСТЯХ ПОСТОЯННОЙ КРИВИЗНЫ}

Рассмотрена квантово-механическая задача двух тел с центральным взаимодействием на односвязных поверхностях постоянной кривизны. При помоши группы изометрий получены системы обыкновенных дифференциальных уравнений для нахождения энергетических уровней. Доказана самосопряженность гамильтониана с несколькими потенциалами взаимодействия, и для сферы в случае равенства масс тел вычислен ряд энергетических серий.

\section{1. ВВЕДЕНИЕ}

С точки зрения теории симметрий пространства постоянной секционной кривизны являются почти столь же естественными кандидатами на роль физического пространства, как евклидово пространство $\mathbf{E}^{n}$, поскольку они, по крайней мере локально, не имеют выделенных точек и направлений. Пространство Лобачевского (гиперболическое пространство) $\mathbf{H}^{n}$, сфера $\mathbf{S}^{n}$ и проективное пространство $\mathbf{S}^{n} /( \pm I)$ являются однородными и изотропными глобально, а также обладают транзитивными группами изометрий максимально возможной размерности $n(n+1) / 2[1]$. Вместе с тем механика на данных пространствах мало изучена, т.к. из-за отсутствия в них аналогов преобразования Галилея уже для задачи двух частиц с центральным взаимодействием переменные не разделяются. Одночастичные задачи на пространствах $\mathbf{H}^{n}$ и $\mathbf{S}^{n}$ изучались в работах [2-11].

В связи с вышесказанным возникает вопрос, в какой мере может быть упрошена задача двух тел при помоши достаточно широкой группы изометрий на пространствах постоянной секционной кривизны? Классическая механическая система двух частиц с центральным взаимодействием была редуцирована в работе [12] с применением метода Марс дена-Вейнстайна к гамильтоновой системе с двумя степенями свободы. В этой работе получены канонически-сопряженные координаты на фазовом пространстве, классифицированы приведенные гамильтонианы, получены условия, обеспечивающие нали-

\footnotetext{
* Московский государственный университет, Москва, Россия. E-mail: alexey@quant.phys.msu.su
} 
чие глобальной динамики. Авторы работ $[13,14]$ попытались перенести метод Марсдена-Вейнстайна на квантовый случай, заменив уровень отображения момента подпространством исходного гильбертова пространства, состоящим из общих собственных функций операторов инфинитезимальных изометрий. Однако, как правило, данное подпространство оказывается слишком узким, если группа изометрий некоммутативна.

В данной работе изучаются квантово-механические двухчастичные задачи с центральным взаимодействием на пространствах $\mathbf{H}^{2}$ и $\mathbf{S}^{2}$. На конфигурационных пространствах этих задач $\mathbf{H}^{2} \times \mathbf{H}^{2}$ и $\mathbf{S}^{2} \times \mathbf{S}^{2}$ группы симметрий действуют свободно, за исключением множества нулевой меры, что позволяет ввести координаты $(r, \theta, \phi, \psi)$, где $r$ - функция расстояния между частицами, а $\theta, \phi, \psi$ - координаты на группе симметрий. С помошью этих координат спектральная задача для двухчастичных гамильтонианов сводится к системам обыкновенных дифференциальных уравнений, каждая из которых соответствует некоторому неприводимому представлению группы изометрий, входяшему в регулярное представление. Для некоторых таких представлений малой размерности в случае пространства $\mathbf{S}^{2} \times \mathbf{S}^{2}$ можно получить отдельные уравнения на собственные функции и для ряда потенциалов взаимодействия точно вычислить серии энергетических уровней. Тем не менее не известен ни один ненулевой потенциал, для которого стационарное уравнение Шредингера для данных систем допускало бы разделение переменных и (или) соответствуюшая классическая задача была бы интегрируемой.

\section{2. ОСНОВНЫЕ ОБОЗНАЧЕНИЯ}

$\mathrm{C}$ целью сократить последуюшие выкладки выберем модели пространств $\mathbf{H}^{2}$ и $\mathbf{S}^{2}$ так, чтобы с помощью формальных замен можно было преобразовывать утверждения, справедливые для одного пространства, в утверждения, справедливые для другого. Пусть сфера $\mathbf{S}^{2}$ реализована в виде расширенной комплексной плоскости $\overline{\mathbf{C}}$ с метрикой

$$
d s^{2}=\frac{4 R^{2}\left(d x^{2}+d y^{2}\right)}{\left(1+x^{2}+y^{2}\right)^{2}}, \quad x+i y=z \in \mathbf{C},
$$

где $R$ - радиус кривизны. В данной модели евклидовы углы совпадают с неевклидовыми. Соответствуюшая метрике (1) мера имеет вид

$$
d \mu=\frac{4 R^{2} d x \wedge d y}{\left(1+x^{2}+y^{2}\right)^{2}} .
$$

Расстояние между двумя точками обозначим $\rho^{s}(\cdot, \cdot)$.

Связная компонента единицы группы изометрий есть группа $\mathrm{SU}(2) /\{ \pm I\} \simeq \mathrm{SO}(3)$, и элемент

$$
\left(\begin{array}{cc}
\alpha & \beta \\
-\bar{\beta} & \bar{\alpha}
\end{array}\right) \in \mathrm{SU}(2), \quad|\alpha|^{2}+|\beta|^{2}=1, \quad \alpha, \beta \in \mathbf{C},
$$

осушествляет отображение

$$
z \longrightarrow \frac{\alpha z+\beta}{-\bar{\beta} z+\bar{\alpha}}
$$


В качестве координат на группе используются углы Эйлера $\theta, \phi, \psi[15]$, при этом

$$
\alpha=\cos \left(\frac{\theta}{2}\right) e^{i \frac{\phi+\psi}{2}}, \quad \beta=i \sin \left(\frac{\theta}{2}\right) e^{i \frac{\phi-\psi}{2}} .
$$

Для группы $\mathrm{SU}(2)$ углы Эйлера изменяются в пределах $0 \leqslant \phi<2 \pi, \quad 0 \leqslant \theta<\pi$, $-2 \pi \leqslant \psi<2 \pi$. Группа $\mathrm{SO}(3)$ двукратно накрывается группой $\mathrm{SU}(2)$, и для нее $0 \leqslant \psi<2 \pi$. Соответствуюшая алгебра Ли отождествляется с левоинвариантными векторными полями на группе, и ее базис имеет вид

$$
\begin{aligned}
& X_{1}=\cos (\psi) \frac{\partial}{\partial \theta}+\frac{\sin (\psi)}{\sin (\theta)} \frac{\partial}{\partial \phi}-\operatorname{ctg}(\theta) \sin (\psi) \frac{\partial}{\partial \psi} \\
& X_{2}=-\sin (\psi) \frac{\partial}{\partial \theta}+\frac{\cos (\psi)}{\sin (\theta)} \frac{\partial}{\partial \phi}-\operatorname{ctg}(\theta) \cos (\psi) \frac{\partial}{\partial \psi} \\
& X_{3}=\frac{\partial}{\partial \psi}
\end{aligned}
$$

с коммутационными соотношениями

$$
\left[X_{p}, X_{j}\right]=\sum_{k=1}^{3} \varepsilon_{p j k} X_{k}, \quad p, j=1,2,3 .
$$

Здесь и далее скобки $[\cdot, \cdot]$ обозначают стандартньй коммутатор векторных полей.

Алгебре инфинитезимальных изометрий на $\mathbf{S}^{2} \times \mathbf{S}^{2}$ (полей Киллинга) соответствуют правоинвариантные векторные поля. Выберем базис $\widetilde{X}_{i}$ этой алгебры так, чтобы $X_{i}$ и $\widetilde{X}_{i}$ переходили друг в друга при замене $\phi \leftrightarrow \psi$ :

$$
\begin{aligned}
& \widetilde{X}_{1}=\cos (\phi) \frac{\partial}{\partial \theta}+\frac{\sin (\phi)}{\sin (\theta)} \frac{\partial}{\partial \psi}-\operatorname{ctg}(\theta) \sin (\phi) \frac{\partial}{\partial \phi} \\
& \widetilde{X}_{2}=-\sin (\phi) \frac{\partial}{\partial \theta}+\frac{\cos (\phi)}{\sin (\theta)} \frac{\partial}{\partial \psi}-\operatorname{ctg}(\theta) \cos (\phi) \frac{\partial}{\partial \phi} \\
& \widetilde{X}_{3}=\frac{\partial}{\partial \phi}
\end{aligned}
$$

где имеют место соотношения

$$
\left[\widetilde{X}_{p}, \widetilde{X}_{j}\right]=\sum_{k=1}^{3} \varepsilon_{p j k} \widetilde{X}_{k}, \quad\left[X_{p}, \widetilde{X}_{j}\right]=0, \quad p, j=1,2,3
$$

Пусть $\mathcal{L}^{2}(G, d \eta)$ - пространство квадратично-интегрируемых на группе функций относительно двухсторонне инвариантной меры $d \eta$. Для групп $\mathrm{SU}(2)$ и $\mathrm{SO}(3)$ данная мера 
имеет вид $d \eta^{s}=\sin \theta d \theta d \phi d \psi$. На этом пространстве группа $G$ действует левыми сдвигами. Известно $[15]^{1)}$, что

$$
\mathcal{L}^{2}(\mathrm{SO}(3))=\bigoplus_{l=0}^{\infty} \bigoplus_{m=-l}^{l} T_{m}^{l}, \quad l, m \in \mathbf{Z}
$$

где $T_{m}^{l}$ - неприводимые представления группы $\mathrm{SO}(3)$, ортогональные друг другу и эквивалентные при одинаковых $l$, причем $\operatorname{dim} T_{m}^{l}=2 l+1$. На пространстве $T_{m}^{l}$ можно выбрать базис $t_{m n}^{l}$ из собственных функций оператора $X_{3}$ так, что

$$
\begin{aligned}
H_{+} t_{m, n}^{l} & =-\sqrt{(l-n)(l+n+1)} t_{m, n+1}^{l}, \\
H_{-} t_{m, n}^{l} & =-\sqrt{(l+n)(l-n+1)} t_{m, n-1}^{l}, \\
H_{3} t_{m, n}^{l} & =n t_{m, n}^{l}, \quad n=-l, \ldots, l,
\end{aligned}
$$

где $H_{ \pm}=i X_{1} \pm X_{2}, H_{3}=i X_{3}$.

Аналогично имеет место разложение

$$
\mathcal{L}^{2}(\mathrm{SO}(3))=\bigoplus_{l=0}^{\infty} \bigoplus_{n=-l}^{l} \widetilde{T}_{n}^{l},
$$

где функции $t_{m n}^{l}, m=-l, \ldots, l$, образуют базис в пространстве $\widetilde{T}_{n}^{l}$ и эти пространства сохраняются операторами $\widetilde{X}_{j}, j=1,2,3$. При этом справедливы формулы, аналогичные формулам (3)-(5):

$$
\begin{aligned}
\widetilde{H}_{+} t_{m, n}^{l} & =-\sqrt{(l-m)(l+m+1)} t_{m+1, n}^{l}, \\
\widetilde{H}_{-} t_{m, n}^{l} & =-\sqrt{(l+m)(l-m+1)} t_{m-1, n}^{l}, \\
\widetilde{H}_{3} t_{m, n}^{l} & =m t_{m, n}^{l}, \quad m=-l, \ldots, l,
\end{aligned}
$$

где $\widetilde{H}_{ \pm}=i \widetilde{X}_{1} \pm \widetilde{X}_{2}, \widetilde{H}_{3}=i \widetilde{X}_{3}$.

На группе $\mathrm{SO}(3)$ имеется двухсторонне инвариантная метрика

$$
d s^{2}=d \psi^{2}+d \phi^{2}+d \theta^{2}+2 \cos \theta d \phi d \psi
$$

относительно которой оператор Лапласа-Бельтрами совпадает с оператором Казимира для алгебры so(3) и имеет вид

$$
K=X_{1}^{2}+X_{2}^{2}+X_{3}^{2}=\widetilde{X}_{1}^{2}+\widetilde{X}_{2}^{2}+\widetilde{X}_{3}^{2}, \quad K t_{m, n}^{l}=-l(l+1) t_{m, n}^{l} .
$$

\footnotetext{
1) Обозначения по сравнению с этой работой несколько изменены с целью большего единообразия гиперболического и сферического случаев.
} 
Для пространства $\mathbf{H}^{2}$ возьмем модель Пуанкаре в единичном круге с метрикой

$$
d s^{2}=\frac{4 R^{2}\left(d x^{2}+d y^{2}\right)}{\left(1-x^{2}-y^{2}\right)^{2}}, \quad x+i y=w \in \mathbf{C}, \quad|w|<1 .
$$

Расстояние между двумя точками обозначим $\rho^{\mathrm{h}}(\cdot, \cdot)$. Связной компонентой единицы группы изометрий является группа $\mathrm{SU}_{\mathrm{f}}(1,1)=\mathrm{SU}(1,1) /\{ \pm I\}$, и элементу

$$
\left(\begin{array}{cc}
\alpha & \beta \\
\bar{\beta} & \bar{\alpha}
\end{array}\right) \in \mathrm{SU}(1,1), \quad|\alpha|^{2}-|\beta|^{2}=1, \quad \alpha, \beta \in \mathbf{C},
$$

соответствует левое действие

$$
z \longrightarrow \frac{\alpha z+\beta}{\bar{\beta} z+\bar{\alpha}}
$$

Параметризация данной группы получается из параметризации группы $\mathrm{SO}(3)$ заменой $\theta \rightarrow-i \tau$. Таким образом, параметры $\alpha$ и $\beta$ имеют вид

$\alpha=\operatorname{ch}\left(\frac{\tau}{2}\right) e^{i \frac{\phi+\psi}{2}}, \quad \beta=i \operatorname{sh}\left(\frac{\tau}{2}\right) e^{i \frac{\phi-\psi}{2}}, \quad 0 \leqslant \phi \leqslant 2 \pi, \quad 0 \leqslant \tau<\infty, \quad 0 \leqslant \psi<2 \pi$.

Данная замена переводит $X_{j}$ в $Y_{j}, j=1,2,3$, где

$$
\begin{aligned}
Y_{1} & =i \cos (\psi) \frac{\partial}{\partial \tau}+i \frac{\sin (\psi)}{\operatorname{sh}(\tau)} \frac{\partial}{\partial \phi}-i \operatorname{cth}(\tau) \sin (\psi) \frac{\partial}{\partial \psi} \\
Y_{2} & =-i \sin (\psi) \frac{\partial}{\partial \tau}+i \frac{\cos (\psi)}{\operatorname{sh}(\tau)} \frac{\partial}{\partial \phi}-i \operatorname{cth}(\tau) \cos (\psi) \frac{\partial}{\partial \psi} \\
X_{3} & =\frac{\partial}{\partial \psi}
\end{aligned}
$$

Операторы $H_{ \pm}, H_{3}$ переходят в операторы $K_{ \pm}=i Y_{1} \pm Y_{2}, K_{3}=i Y_{3}$, соответственно.

Двухсторонне инвариантная мера на группе $\mathrm{SU}_{\mathrm{f}}(1,1)$ имеет вид $d \eta^{\mathrm{h}}=\operatorname{sh} \tau d \tau d \phi d \psi$, а пространство $\mathcal{L}^{2}\left(\mathrm{SU}_{\mathrm{f}}(1,1), d \eta^{\mathrm{h}}\right)$ регулярного представления разлагается следуюшим образом [15]:

$$
\begin{aligned}
& \mathcal{L}^{2}\left(\mathrm{SU}_{\mathrm{f}}(1,1), d \eta^{\mathrm{h}}\right)= {\left[\bigoplus_{m=-\infty}^{\infty} \int_{0}^{\infty} H_{-\frac{1}{2}+i \rho}^{m} \rho \operatorname{th}(\pi \rho) d \rho\right] \oplus } \\
& \oplus\left[\bigoplus_{\substack{m=-\infty \\
m \neq 0}}^{\infty} \bigoplus_{l=0}^{|m|-1}\left(l+\frac{1}{2}\right) H_{l}^{m}\right] .
\end{aligned}
$$

Пространство $H_{-\frac{1}{2}+i \rho}^{m}, \rho \in \mathbf{R}$, имеет базис

$$
h_{m, n}^{\left(-\frac{1}{2}+i \rho\right)}(\tau, \phi, \psi)=e^{-i(m \phi+n \psi)} \mathcal{B}_{m, n}^{\left(-\frac{1}{2}+i \rho\right)}(\operatorname{ch} \tau), \quad n=-\infty, \ldots, \infty
$$


где

$$
\begin{aligned}
\mathcal{B}_{m, n}^{\chi}(\operatorname{ch} \tau)= & \frac{1}{2 \pi} \int_{0}^{2 \pi}\left(\operatorname{ch}\left(\frac{\tau}{2}\right)+\operatorname{sh}\left(\frac{\tau}{2}\right) e^{i \theta}\right)^{\chi+n} \times \\
& \times\left(\operatorname{ch}\left(\frac{\tau}{2}\right)+\operatorname{sh}\left(\frac{\tau}{2}\right) e^{-i \theta}\right)^{\chi-n} e^{i(m-n) \theta} d \theta, \quad 0 \leqslant \tau<\infty .
\end{aligned}
$$

Пространство $H_{l}^{m}, 0 \leqslant l \leqslant|m|-1$, имеет базис

$$
\left\{h_{m, n}^{(l)}\right\}_{n=l+1}^{\infty} \text { при } m>0, \quad\left\{h_{m, n}^{(l)}\right\}_{n=-l-1}^{-\infty} \text { при } m<0 .
$$

При одинаковых $l$ и разных $m$ эти пространства соответствуют эквивалентным представлениям. Разложение (10) означает, что произвольная функция

$$
f(\tau, \phi, \psi) \in \mathcal{L}^{2}\left(\mathrm{SU}_{\mathrm{f}}(1,1), d \eta^{\mathrm{h}}\right)
$$

представляется в виде

$$
\begin{aligned}
f= & \sum_{m, n=-\infty}^{\infty}\left(\int_{0}^{\infty} a_{m, n}(\rho) h_{m, n}^{\left(-\frac{1}{2}+i \rho\right)}(\tau, \phi, \psi) \rho \operatorname{th}(\pi \rho) d \rho+\right. \\
& \left.+\sum_{l=0}^{M-1}\left(l+\frac{1}{2}\right) b_{m, n}^{l} h_{m, n}^{(l)}(\tau, \phi, \psi)\right),
\end{aligned}
$$

где

$$
M= \begin{cases}\min \{|m|,|n|\} & \text { при } m n \geqslant 0, \\ 0 & \text { при } m n<0 .\end{cases}
$$

Операторы $K_{ \pm}, K_{3}$ действуют на базисные элементы следуюшим образом:

$$
\begin{aligned}
& K_{+} h_{m, n}^{(\chi)}=(n-\chi) h_{m, n+1}^{(\chi)}, \\
& K_{-} h_{m, n}^{(\chi)}=-(n+\chi) h_{m, n-1}^{(\chi)} \\
& K_{3} h_{m, n}^{(\chi)}=n h_{m, n}^{(\chi)}, \quad \chi=-\frac{1}{2}+i \rho, \quad 0 \leqslant \rho<\infty \text { или } \chi=l \in \mathrm{Z} .
\end{aligned}
$$

При этом следует учесть, что в соответствии с данным определением $h_{m, l}^{(l)}=0$ при $m>l \geqslant 0$ и $h_{m,-l}^{(l)}=0$ при $-m>l \geqslant 0$. 


\section{3. ЗАДАЧА ДВУХ ТЕЛ НА СФЕРЕ $\mathrm{S}^{2}$}

Рассмотрим гамильтониан, соответствуюший взаимодействию двух частиц с массами $m_{1}$ и $m_{2}$ на произведении двух экземпляров сфферы $\mathbf{S}^{2}$ с одинаковым радиусом кривизны,

$$
\widehat{H}_{\mathrm{S}}=-\frac{1}{2 m_{1}} \Delta_{1}-\frac{1}{2 m_{2}} \Delta_{2}+U\left(\rho^{\mathrm{s}}(1,2)\right)
$$

где $\Delta_{j}$ - оператор Лапласа-Бельтрами на $\mathbf{S}_{j}^{2}, j=1,2, \rho^{\mathrm{s}}(1,2)$ - риманово расстояние между первой и второй частицами, $U$ - потенциал взаимодействия. Пусть $x_{j}, y_{j}-$ координаты на $\mathbf{S}_{j}^{2}, j=1,2$,

$$
\begin{gathered}
r=\operatorname{tg}\left(\rho^{\mathrm{s}}(1,2) /(2 R)\right), \\
r_{1}=\operatorname{tg}\left(\frac{m_{2}}{m_{1}+m_{2}} \operatorname{tg}^{-1}(r)\right), \quad r_{2}=-\operatorname{tg}\left(\frac{m_{1}}{m_{1}+m_{2}} \operatorname{tg}^{-1}(r)\right),
\end{gathered}
$$

$\mathcal{H}=\mathcal{L}^{2}\left(\mathbf{S}_{1}^{2} \otimes \mathbf{S}_{2}^{2}, d \mu_{1} \otimes d \mu_{2}\right)$ - гильбертово пространство состояний, где $d \mu_{j}, j=1,2,-$ мера на $\mathbf{S}_{j}^{2}$, соответствующая метрике (1). Поскольку элементы группы $\mathrm{SO}(3)$, действуя на $\mathbf{S}_{1}^{2} \otimes \mathbf{S}_{2}^{2}$, могут оставлять неподвижными лишь совпадаюшие или диаметрально противоположные точки, то соответствие между величинами $x_{1}, y_{1}, x_{2}, y_{2}$ и $r, \theta, \phi, \psi$ не является взаимно однозначным лишь при $r=0$ и $r=\infty$, т.е. на множестве нулевой меры. Перейдем к системе координат $r, \theta, \phi, \psi$. Будем считать, что точка пространства $\mathbf{S}_{1}^{2} \otimes \mathbf{S}_{2}^{2}$ с координатами $r, \theta, \phi, \psi$ получается из точки $\left(r_{1}, r_{2}\right) \in \overline{\mathbf{C}} \times \overline{\mathbf{C}}$ под действием элемента группы $\mathrm{SO}(3)$, отвечающего координатам $\theta, \phi, \psi$. Здесь и далее мы опускаем соответствуюшие прямые, иногда очень громоздкие, выкладки, которые были выполнены с использованием системы аналитических компьютерных вычислений Maple V [16].

Метрика

$$
d s^{2}=2 m_{1} d s_{1}^{2}+2 m_{2} d s_{2}^{2}
$$

где

$$
d s_{j}^{2}=\frac{4 R^{2}\left(d x_{j}^{2}+d y_{j}^{2}\right)}{\left(1+x_{j}^{2}+y_{j}^{2}\right)^{2}}, \quad j=1,2,
$$

соответствует оператору Лапласа-Бельтрами $\left(\Delta_{1} / 2 m_{1}\right)+\left(\Delta_{2} / 2 m_{2}\right)$. В координатах $r$, $\theta, \phi, \psi$ она записьвается в виде

$$
d s^{2}=\sum_{j=1}^{2} 2 m_{j} R^{2}\left(\frac{4 d r_{j}^{2}}{\left(1+r_{j}^{2}\right)^{2}}+\frac{1+r_{j}^{4}}{4} d \gamma_{1}^{2}+r_{j}\left(1-r_{j}^{2}\right) d \gamma_{2}^{2}+r_{j}^{2} d \gamma_{3}^{2}\right),
$$

где

$$
\begin{aligned}
d \gamma_{1}^{2}= & d \theta^{2}+\sin ^{2} \theta d \phi^{2} \\
d \gamma_{2}^{2}= & \cos \theta \sin \theta \sin \psi d \phi^{2}+\sin \theta \sin \psi d \psi d \phi+\cos \psi d \psi d \theta+\cos \theta \cos \psi d \phi d \theta \\
d \gamma_{3}^{2}= & d \psi^{2}-\frac{1}{2}(\cos 2 \psi) d \theta^{2}+\left(\cos ^{2} \theta+\frac{1}{2}(\cos 2 \psi) \sin ^{2} \theta\right) d \phi^{2}- \\
& -\sin \theta \sin (2 \psi) d \theta d \phi+2 \cos \theta d \psi d \phi
\end{aligned}
$$


При этом $L_{\tilde{X}_{j}}\left(d \gamma_{k}^{2}\right)=0, j, k=1,2,3$, где $L_{X}(\cdot)$ - производная Ли вдоль векторного поля $X$. Мы видим, что в метрике нет перекрестных членов между $d r$ и групповыми координатами, и то же самое поэтому имеет место для оператора $\widehat{H}_{\mathrm{S}}$.

Mepa $d \mu=d \mu_{1} \otimes d \mu_{2}$ в координатах $r, \theta, \phi, \psi$ имеет вид $d \mu=d \nu \wedge d \eta^{\mathrm{s}}$, где

$$
d \nu=\frac{4 R^{4} r d r}{\left(1+r^{2}\right)^{2}}
$$

Гильбертово пространство состояний $\mathcal{H}$ допускает разложение

$$
\mathcal{H}=\mathcal{L}^{2}\left(\mathbf{R}_{+}, d \nu\right) \otimes \mathcal{L}^{2}\left(\mathrm{SO}(3), d \eta^{\mathrm{s}}\right)=\bigoplus_{l=0}^{\infty} \bigoplus_{m=-l}^{l} \mathcal{H}_{m}^{l}
$$

где прямые суммы ортогональны и

$$
\mathcal{H}_{m}^{l}=\mathcal{L}^{2}\left(\mathbf{R}_{+}, d \nu\right) \otimes T_{m}^{l}
$$

Вследствие равенств $\left[\widehat{H}_{\mathrm{S}}, K\right]=\left[\widehat{H}_{\mathrm{S}}, \widetilde{H}_{3}\right]=0$ оператор $\widehat{H}_{\mathrm{S}}$ действует на пространствах $\mathcal{H}_{m}^{l}$, являюшихся максимальными пространствами собственных функций операторов $K$ и $\widetilde{H}_{3}$ с собственными значениями $-l(l+1)$ и $m$, соответственно. Таким образом, задача на собственные значения для оператора $\widehat{H}_{\mathrm{s}}$ сводится к задачам на собственные значения для его ограничений $\left.\widehat{H}_{\mathrm{s}}\right|_{\mathcal{H}_{m}^{l}}$. При этом, поскольку $\operatorname{dim} T_{m}^{l}=$ $2 l+1$, задача на собственные значения для оператора $\left.\widehat{H}_{\mathrm{S}}\right|_{\mathcal{H}_{m}^{l}}$ состоит в решении системы $2 l+1$ обыкновенных дифференциальных уравнений в пространстве $\mathcal{L}^{2}\left(\mathbf{R}_{+}, d \nu\right)$. Данный подход в некотором смысле обобщает метод разделения переменных.

Гамильтониан $\widehat{H}_{\mathrm{S}}$ в координатах $r, \theta, \phi, \psi$ записывается в виде

$$
\begin{aligned}
\widehat{H}_{\mathrm{S}}= & -\frac{\left(1+r^{2}\right)^{2}}{8 m R^{2}}\left(\frac{1}{r} \frac{\partial}{\partial r}\left(r \frac{\partial}{\partial r}\right)\right)-\frac{1}{2 R^{2}} C(r) X_{1}^{2}-\frac{1}{2 R^{2}} A(r) X_{3}^{2}- \\
& -\frac{1}{8 R^{2}} B(r)\left\{X_{1}, X_{3}\right\}-\frac{1}{2\left(m_{1}+m_{2}\right) R^{2}} K+U(r),
\end{aligned}
$$

где

$$
\begin{aligned}
A(r) & =\frac{\left(1+r^{2}\right)^{2}}{8 m r^{2}}-\frac{1}{m_{1}+m_{2}}+\frac{1-r^{4}}{8 m r^{2}} \cos \zeta+\frac{1+r^{2}}{4 m_{1} m_{2} r}\left(m_{1}-m_{2}\right) \sin \zeta, \\
B(r) & =\frac{\left(m_{2}-m_{1}\right)}{m_{1} m_{2} r}\left(1+r^{2}\right) \cos \zeta+\frac{1-r^{4}}{2 m r^{2}} \sin \zeta, \\
C(r) & =\frac{\left(1+r^{2}\right)^{2}}{8 m r^{2}}-\frac{1}{m_{1}+m_{2}}-\frac{1-r^{4}}{8 m r^{2}} \cos \zeta-\frac{1+r^{2}}{4 m_{1} m_{2} r}\left(m_{1}-m_{2}\right) \sin \zeta, \\
\zeta & =2 \frac{m_{1}-m_{2}}{m_{1}+m_{2}} \operatorname{arctg} r, \quad m=\frac{m_{1} m_{2}}{m_{1}+m_{2}},
\end{aligned}
$$


a $\{\cdot, \cdot\}$ обозначает антикоммутатор. Функции $A(r), B(r), C(r)$ те же, что и в работе [12]. Уравнение на собственные функции

$$
\Psi=\sum_{n=-l}^{l} c^{n}(r) t_{m, n}^{l}
$$

для оператора $\left.\widehat{H}_{\mathrm{s}}\right|_{\mathcal{H}_{m}^{l}}$ имеет вид

$$
\sum_{n=-l}^{l}\left(\widehat{H}_{\mathrm{S}}\left(c^{n}(r) t_{m, n}^{l}\right)-E c^{n}(r) t_{m, n}^{l}\right)=0 .
$$

Каждый уровень энергии, найденный таким образом, является по крайней мере $(2 l+1)$-кратным уровнем для гамильтониана $\widehat{H}_{\mathrm{s}}$, что соответствует различным значениям $m=-l, \ldots, l$. В случае $l=0$ собственная функция $\Psi$ зависит лишь от переменной $r$ и уравнение для нее

$$
-\frac{\left(1+r^{2}\right)^{2}}{8 m R^{2}}\left(\frac{1}{r} \frac{\partial}{\partial r}\left(r \frac{\partial \Psi}{\partial r}\right)\right)+(U(r)-E) \Psi=0
$$

совпадает с соответствуюшим уравнением для одной частицы приведенной массы $m$ в поле силового центра.

Особенно простой вид гамильтониан принимает в случае частиц одинаковой массы $m_{1}=m_{2}=2 m$ :

$$
\widehat{H}_{\mathrm{S}}=L^{\mathrm{S}}+\frac{r^{2}}{32 m R^{2}}\left[H_{+}^{2}+H_{-}^{2}+\left\{H_{+}, H_{-}\right\}\right]+\frac{1}{8 m r^{2} R^{2}} H_{3}^{2}-\frac{1}{8 m R^{2}} K+U(r),
$$

где

$$
L^{\mathrm{S}}=-\frac{\left(1+r^{2}\right)^{2}}{8 m R^{2}}\left(\frac{1}{r} \frac{\partial}{\partial r}\left(r \frac{\partial}{\partial r}\right)\right) .
$$

Уравнение (11) эквивалентно системе уравнений

$$
\begin{gathered}
L\left(c^{n}\right)+\left(\frac{1}{8 m R^{2}}\left(\frac{n^{2}}{r^{2}}+l(l+1)+\frac{r^{2}}{2}\left(l^{2}+l-n^{2}\right)\right)+U-E\right) c^{n}+ \\
+\frac{r^{2}}{32 m R^{2}}\left(q_{n} c^{n-2}+q_{-n} c^{n+2}\right)=0, \quad n=-l, \ldots, l,
\end{gathered}
$$

где

$$
q_{n}=\sqrt{(l-n+2)\left(l^{2}-(n-1)^{2}\right)(l+n)} .
$$

Вводя переменные $c_{ \pm}^{n}=c^{n} \pm c^{-n}$, получаем, что система (13) распадается на четыре независимые системы:

$$
\begin{aligned}
L\left(c_{ \pm}^{n}\right)+ & \left(\frac{1}{8 m R^{2}}\left(\frac{n^{2}}{r^{2}}+l(l+1)+\frac{r^{2}}{2}\left(l^{2}+l-n^{2}\right)\right)+U-E\right) c_{ \pm}^{n}+ \\
& +\frac{r^{2}}{32 m R^{2}}\left(q_{n} c_{ \pm}^{n-2}+q_{-n} c_{ \pm}^{n+2}\right)=0, \quad n=0, \ldots, l
\end{aligned}
$$


где выбирается либо плюс, либо минус, а $n$ пробегает либо четные, либо нечетные значения. С учетом соотношений $c_{+}^{n}=c_{+}^{-n}, c_{-}^{n}=-c_{-}^{-n}, c_{-}^{0}=0$ мы видим, что, кроме случая $l=n=0$, указанные системы состоят из одного уравнения в следуюших семи случаях:

a) $l=1$ :

$$
\begin{aligned}
L\left(c_{+}^{0}\right)+\left(\frac{1}{8 m R^{2}}\left(2+r^{2}\right)+U-E\right) c_{+}^{0} & =0, \\
L\left(c_{+}^{1}\right)+\left(\frac{1}{8 m R^{2}}\left(\frac{1}{r^{2}}+2+r^{2}\right)+U-E\right) c_{+}^{1} & =0, \\
L\left(c_{-}^{1}\right)+\left(\frac{1}{8 m R^{2}}\left(\frac{1}{r^{2}}+2\right)+U-E\right) c_{-}^{1} & =0 ;
\end{aligned}
$$

б) $l=2$ :

$$
\begin{gathered}
L\left(c_{-}^{2}\right)+\left(\frac{1}{8 m R^{2}}\left(\frac{4}{r^{2}}+6+r^{2}\right)+U-E\right) c_{-}^{2}=0 \\
L\left(c_{-}^{1}\right)+\left(\frac{1}{8 m R^{2}}\left(\frac{1}{r^{2}}+6+r^{2}\right)+U-E\right) c_{-}^{1}=0 \\
L\left(c_{+}^{1}\right)+\left(\frac{1}{8 m R^{2}}\left(\frac{1}{r^{2}}+6+4 r^{2}\right)+U-E\right) c_{+}^{1}=0
\end{gathered}
$$

в) $l=3$ :

$$
L\left(c_{-}^{2}\right)+\left(\frac{1}{2 m R^{2}}\left(\frac{1}{r^{2}}+3+r^{2}\right)+U-E\right) c_{-}^{2}=0 .
$$

Заметим, что уравнение для радиальной компоненты $c(r)$ собственной функции одной частицы в центральном поле имеет вид

$$
L(c)+\left(\frac{n^{2}}{8 m R^{2}}\left(\frac{1}{r^{2}}+2+r^{2}\right)+U-E\right) c=0, \quad n=0,1,2 \ldots
$$

Поэтому собственные значения из уравнений (12), (15), (18), (20) вычисляются точно всегда, когда они вычисляются точно из уравнения $(21)$ при любом $n \in \mathbf{R}$. С другой стороны, при $U \equiv 0$ мы имеем систему двух невзаимодействуюших частиц, спектр которой вычисляется точно. Уравнение

$$
-\mu \frac{\left(1+r^{2}\right)^{2}}{4 r} \frac{\partial}{\partial r}\left(r \frac{\partial c}{\partial r}\right)+\left(\eta r^{-2}+\nu r^{2}-E\right) c=0, \quad \mu, \eta, \nu=\text { const }
$$

относительно переменной $\xi=r^{2}$ становится уравнением Римана с особыми точками $0,-1, \infty[17]$

$$
\frac{d^{2} c}{d \xi^{2}}+\frac{1}{\xi} \frac{d c}{d \xi}-\frac{\eta+\nu \xi^{2}-E \xi}{\mu \xi^{2}(1+\xi)^{2}} c=0,
$$

которое, в свою очередь, сводится к гипергеометрическому уравнению.

4 Теоретическая и математическая физика, т. 118, № 2, 1999 г. 
Таким образом, уравнения (14)-(20) сводятся к гипергеометрическому уравнению при $U(r)=a r^{-2}+b r^{2}, a, b=$ const. Соответствуюшие собственные значения вычисляются точно, если задать область определения $D\left(\widehat{H}_{\mathrm{S}}\right)$, например, так, чтобы оператор $\widehat{H}_{\mathrm{s}}$ был самосопряженным.

На сфере $\mathbf{S}^{n}$ известны аналоги кулоновского и осцилляторного потенциалов [4]:

$$
U_{\mathrm{q}}=\frac{\kappa}{2 R}\left(r-\frac{1}{r}\right), \quad U_{\mathrm{o}}=\frac{2 \omega^{2} R^{2} r^{2}}{\left(1-r^{2}\right)^{2}} .
$$

Они замечательны тем, что все траектории классического одночастичного движения в этих потенциалах замкнуты. Радиальное уравнение одночастичной спектральной задачи для этих потенциалов сводится к уравнению Римана относительно независимых переменных $u=\left(1-r^{2}\right) / r$ и $v=u^{2}$, соответственно [11]. Однако коэффициенты уравнения (22) рациональны относительно переменных $u$ или $v$ лиш при $\eta=\nu$, что не дает ничего нового, т.к. в этом случае оно является частным случаем уравнения (21).

Полуограниченные симметричные операторы всегда имеют самосопряженное расширение по Фридрихсу [18]. При этом в качестве начальной области определения удобно выбрать множество гладких финитных на подходящей области функций. Однако для вычисления спектра оператора необходимо точное описание области определения этого расширения. Для потенциалов $U \geqslant 0, U \in \mathcal{L}_{\mathrm{loc}}^{1}\left(\mathbf{R}^{n}\right)$, область определения расширения по Фридрихсу оператора $-\Delta+U$ на пространстве $\mathbf{R}^{n}$ имеет вид [18]

$$
D(-\Delta+U)=\left\{\phi \in \mathcal{L}^{2}\left(\mathbf{R}^{n}\right) \mid V \phi \in \mathcal{L}_{\text {loc }}^{1}\left(\mathbf{R}^{n}\right), \quad(-\Delta \phi+V \phi)_{\text {oф }} \in \mathcal{L}^{2}\left(\mathbf{R}^{n}\right)\right\},
$$

где “оф” означает обобшенную функцию. Как уже отмечалось в работе [11], данный результат полностью переносится на полные римановы пространства, если под суммируемостью функций понимать суммируемость относительно меры, порожденной метрикой. Для неотрицательных потенциалов, не принадлежащих множеству $\mathcal{L}_{\text {loc }}^{1}$, типа потенциалов $a r^{-2}+b r^{2}, a, b \geqslant 0$, и $U_{\mathrm{o}}$, утверждение об области определения самосопряженного по Фридрихсу расширения становится справедливым, если ограничиться той областью пространства, в которой потенциал $V \in \mathcal{L}_{\text {loc }}^{1}$. Таким образом, справедлива следующая теорема.

ТЕОРЕМА. Самосопряженное расширение по Фридрихсу двухчастичного гамильтониана на сфере $\mathbf{S}^{2}$

$$
\widehat{H}_{\mathrm{s}}^{(1)}=-\frac{\Delta_{1}}{2 m_{1}}-\frac{\Delta_{2}}{2 m_{2}}+U_{\mathrm{o}}
$$

имеет область определения

$$
D\left(\widehat{H}_{\mathrm{s}}^{(1)}\right)=\left\{\phi \in \mathcal{L}^{2}(M, d \mu) \mid U_{\mathrm{o}} \phi \in \mathcal{L}_{\text {loc }}^{1}(M, d \mu), \quad\left(\widehat{H}^{(1)}(\phi)\right)_{\mathrm{o \phi}} \in \mathcal{L}^{2}(M, d \mu)\right\},
$$

где $M$ - одна из двух связных компонент мнохества

$$
\left\{(x, y) \in \mathbf{S}^{2} \times \mathbf{S}^{2} \mid \rho(x, y)<\frac{\pi R}{2}\right\} .
$$


Аналогичное расширение для гамильтониана

$$
\widehat{H}_{\mathrm{s}}^{(2)}=-\frac{\Delta_{1}}{2 m_{1}}-\frac{\Delta_{2}}{2 m_{2}}+a r^{2}+b r^{-2}, \quad a, b \geqslant 0
$$

имеет область определения, задаваемую формулой (23), әде $M=\mathbf{S}^{2} \times \mathbf{S}^{2}$. Действия расширений на этих областях определения понимаются в смысле обобщенных функиий.

Энергетические уровни из уравнений $(12),(14)-(20)$ находятся прямым сравнением с энергетическими уровнями для уравнения (21) [4]:

$$
E_{k, n}=\frac{(n+2 k+1)^{2}}{2 R^{2} m}+\frac{\omega(n+2 k+1)}{\sqrt{m}} \sqrt{1+\frac{1}{4 \omega^{2} R^{4} m}}, \quad k=0,1,2 \ldots,
$$

что приводит к следующим энергетическим уровням для уравнений (12), (15), (18), (20) $(k=0,1,2, \ldots)$ :

$$
\begin{aligned}
& E_{k}^{(0)}=\frac{(2 k+1)^{2}}{2 R^{2} m}+\frac{\omega(2 k+1)}{\sqrt{m}} \sqrt{1+\frac{1}{4 \omega^{2} R^{4} m}}, \\
& E_{k}^{(1)}=\frac{2(k+1)^{2}}{R^{2} m}+\frac{2 \omega(k+1)}{\sqrt{m}} \sqrt{1+\frac{1}{4 \omega^{2} R^{4} m}}, \\
& E_{k}^{(2)}=E_{k}^{(1)}+\frac{1}{2 m R^{2}}, \\
& E_{k}^{(3)}=E_{k+1}^{(0)}+\frac{1}{2 m R^{2}},
\end{aligned}
$$

соответственно. Таким образом, точно вычисляются по крайней мере четыре серии уровней энергии для двухчастичной квантовой системы с равными массами для осцилляторного потенциала на $\mathbf{S}^{2}$.

Энергетические уровни для уравнения (22)

$$
E_{k}=(2 k+1)(\sqrt{\mu \nu}+\sqrt{\mu \eta})+k(k+1) \mu+2 \sqrt{\eta \nu}, \quad k=0,1,2 \ldots
$$

приводят к энергетическим уровням для уравнений (12), (14)-(20) при $U=a r^{-2}+b r^{2}$ $(k=0,1,2, \ldots)$ :

$$
\begin{aligned}
\widetilde{E}_{k}^{(0,1)}(a, b)= & \frac{2 k+1}{\sqrt{2 m} R}(\sqrt{a}+\sqrt{b})+\frac{k(k+1)}{2 m R^{2}}+2 \sqrt{a b} \\
\widetilde{E}_{k}^{(1,1)}(a, b)= & \frac{1}{4 m R^{2}}\left(1+(2 k+1) \sqrt{1+8 m R^{2} b}\right)+ \\
& +\frac{k(k+1)}{2 m R^{2}}+\frac{2 k+1}{R} \sqrt{\frac{a}{2 m}}+\sqrt{\frac{a}{2 m R^{2}}+4 a b} \\
\widetilde{E}_{k}^{(1,2)}(a, b)= & \frac{1}{4 m R^{2}}+\frac{2 k+1}{\sqrt{2 m} R}\left(\sqrt{a+\frac{1}{8 m R^{2}}}+\sqrt{b+\frac{1}{8 m R^{2}}}\right)+\frac{k(k+1)}{2 m R^{2}}+
\end{aligned}
$$




$$
\begin{aligned}
& +\sqrt{\left(\frac{1}{4 m R^{2}}+2 a\right)\left(\frac{1}{4 m R^{2}}+2 b\right)}, \\
\widetilde{E}_{k}^{(1,3)}(a, b)= & \widetilde{E}_{k}^{(1,1)}(b, a), \\
\widetilde{E}_{k}^{(2,1)}(a, b)= & \frac{3}{4 m R^{2}}+\frac{2 k+1}{\sqrt{2 m} R}\left(\sqrt{a+\frac{1}{2 m R^{2}}}+\sqrt{\left.b+\frac{1}{8 m R^{2}}\right)}+\frac{k(k+1)}{2 m R^{2}}+\right. \\
& +\sqrt{\left(\frac{1}{m R^{2}}+2 a\right)\left(\frac{1}{4 m R^{2}}+2 b\right)}, \\
\widetilde{E}_{k}^{(2,2)}(a, b)= & \widetilde{E}_{k}^{(1,2)}(b, a)+\frac{1}{2 m R^{2}}, \\
\widetilde{E}_{k}^{(2,3)}(a, b)= & \widetilde{E}_{k}^{(2,1)}(b, a), \\
\widetilde{E}_{k}^{(3,1)}(a, b)= & \frac{3}{4 m R^{2}}+\frac{2 k+1}{\sqrt{2 m} R}\left(\sqrt{a+\frac{1}{2 m R^{2}}}+\sqrt{b+\frac{1}{2 m R^{2}}}\right)+\frac{k(k+1)}{2 m R^{2}}+ \\
& +\sqrt{\left(\frac{1}{m R^{2}}+2 a\right)\left(\frac{1}{m R^{2}}+2 b\right)},
\end{aligned}
$$

соответственно.

Заметим, что в соответствии со сказанным выше серии $E_{k}^{(l)}$ и $\widetilde{E}^{(l, \cdot)}, l=0,1,2,3$, состоят по крайней мере из $(2 l+1)$-кратно вырожденных энергетических уровней.

\section{4. ЗАДАЧА ДВУХ ТЕЛ НА ПРОСТРАНСТВЕ Н}

Заметим, что формальная замена переменных $R \rightarrow i R, x \rightarrow i x, y \rightarrow i y, r \rightarrow i r$, $r_{1,2} \rightarrow i r_{1,2}, H_{ \pm}^{l} \rightarrow K_{ \pm}^{l}, \theta \rightarrow-i \tau$ переводит формулы, справедливые для пространства $\mathbf{S}^{2}$, в формулы, справедливые для пространства $\mathbf{H}^{2}$. Поэтому мы получаем следующие результаты, не повторяя длинных вычислений.

Теперь мы имеем

$$
\begin{gathered}
r=\operatorname{th}\left(\frac{\rho^{\mathrm{h}}(1,2)}{2 R}\right), \quad 0 \leqslant \rho^{\mathrm{h}}(1,2)<\infty, \\
\mathcal{H}=\mathcal{L}^{2}\left(\mathbf{H}_{1}^{2} \otimes \mathbf{H}_{2}^{2}, d \mu_{1} \otimes d \mu_{2}\right), \quad d \mu_{1} \otimes d \mu_{2}=d \nu \wedge d \eta^{\mathrm{h}},
\end{gathered}
$$

где

$$
d \nu=\frac{4 R^{4}\left|1-r_{1} r_{2}\right|\left|r_{1}-r_{2}\right| d r}{\left(1-r^{2}\right)\left(1-r_{1}^{2}\right)\left(1-r_{2}^{2}\right)},
$$

a $d \mu_{i}$ - мера на пространстве $\mathbf{H}^{2}$, соответствуюшая метрике (9).

Гильбертово пространство состояний $\mathcal{H}$ допускает разложение

$$
\begin{aligned}
\mathcal{H} & =\mathcal{L}^{2}\left(\mathbf{R}_{+}, d \nu\right) \otimes \mathcal{L}^{2}\left(\operatorname{SU}_{\mathrm{f}}(1,1), d \eta^{\mathrm{h}}\right)= \\
& =\left[\bigoplus_{m=-\infty}^{\infty} \int_{0}^{\infty} \mathcal{H}_{\rho}^{m} \rho \operatorname{th}(\pi \rho) d \rho\right] \oplus\left[\bigoplus_{\substack{m=-\infty \\
m \neq 0}}^{\infty} \bigoplus_{l=0}^{|m|-1}\left(l+\frac{1}{2}\right) \mathcal{H}_{l}^{m}\right],
\end{aligned}
$$


где

$$
\mathcal{H}_{\rho}^{m}=\mathcal{L}^{2}\left(\mathbf{R}_{+}, d \nu\right) \otimes H_{-\frac{1}{2}+i \rho}^{m}, \quad \mathcal{H}_{l}^{m}=\mathcal{L}^{2}\left(\mathbf{R}_{+}, d \nu\right) \otimes H_{l}^{m}
$$

Двухчастичный гамильтониан $\widehat{H}_{\mathrm{h}}$ принимает теперь вид

$$
\begin{aligned}
\widehat{H}_{\mathrm{h}}= & -\frac{\left(1-r^{2}\right)^{2}}{8 m R^{2}}\left(\frac{1}{r} \frac{\partial}{\partial r}\left(r \frac{\partial}{\partial r}\right)\right)-\frac{1}{2 R^{2}} C(r) Y_{1}^{2}-\frac{1}{2 R^{2}} A(r) Y_{3}^{2}- \\
& -\frac{i}{8 R^{2}} B(r)\left\{Y_{1}, Y_{3}\right\}+\frac{1}{2\left(m_{1}+m_{2}\right) R^{2}} K+U(r),
\end{aligned}
$$

где

$$
\begin{aligned}
A(r) & =\frac{\left(1-r^{2}\right)^{2}}{8 m r^{2}}+\frac{1}{m_{1}+m_{2}}+\frac{1-r^{4}}{8 m r^{2}} \operatorname{ch} \zeta-\frac{1-r^{2}}{4 m_{1} m_{2} r}\left(m_{1}-m_{2}\right) \operatorname{sh} \zeta, \\
B(r) & =\frac{\left(m_{2}-m_{1}\right)}{m_{1} m_{2} r}\left(1-r^{2}\right) \operatorname{ch} \zeta+\frac{1-r^{4}}{2 m r^{2}} \operatorname{sh} \zeta, \\
C(r) & =\frac{\left(1-r^{2}\right)^{2}}{8 m r^{2}}+\frac{1}{m_{1}+m_{2}}-\frac{1-r^{4}}{8 m r^{2}} \operatorname{ch} \zeta+\frac{1-r^{2}}{4 m_{1} m_{2} r}\left(m_{1}-m_{2}\right) \operatorname{sh} \zeta, \\
\zeta & =2 \frac{m_{1}-m_{2}}{m_{1}+m_{2}} \operatorname{arcth} r .
\end{aligned}
$$

В случае $m_{1}=m_{2}=2 m$ гамильтониан записывается в виде

$$
\widehat{H}_{\mathrm{h}}=L^{\mathrm{h}}+\frac{r^{2}}{32 m R^{2}}\left[K_{+}^{2}+K_{-}^{2}+\left\{K_{+}, K_{-}\right\}\right]+\frac{1}{8 m r^{2} R^{2}} K_{3}^{2}+\frac{1}{8 m R^{2}} K+U(r),
$$

где

$$
L^{\mathrm{h}}=-\frac{\left(1-r^{2}\right)^{2}}{8 m R^{2}}\left(\frac{1}{r} \frac{\partial}{\partial r}\left(r \frac{\partial}{\partial r}\right)\right)
$$

При ограничении данного оператора $\widehat{H}_{\mathrm{h}}$ на инвариантные подпространства $\mathcal{H}_{\rho}^{m}$ и $\mathcal{H}_{l}$ соответствующие системы уравнений на собственные значения обладают теми же симметриями, что и в сферическом случае. Однако каждое из инвариантных пространств бесконечномерно и отдельных уравнений на собственные значения не возникает. По-видимому, двухчастичный гамильтониан на пространстве $\mathbf{H}^{n}$ не имеет дискретного спектра. Косвенным подтверждением этого является отсутствие дискретного спектра у двухчастичного гамильтониана в евклидовом пространстве с центральным взаимодействием, если не производить отделения его части, соответствующей движению центра масс. Об этом факте почему-то умалчивают учебники по квантовой механике. Действительно, двухчастичный гамильтониан в евклидовом пространстве $\mathbf{E}^{n}$ заменой переменных приводится к виду [19]

$$
\widehat{H}=\widehat{H}_{\mathrm{r}}+\widehat{H}_{\mathrm{c}}, \quad \widehat{H}_{\mathrm{r}}=-\frac{1}{2 m} \Delta_{\mathrm{r}}+U, \quad \widehat{H}_{\mathrm{c}}=-\frac{1}{2 m} \Delta_{\mathrm{c}}
$$


где $\Delta_{\mathrm{r}}$ - лапласиан, отвечающий относительному движению частиц, а лапласиан $\Delta_{\mathrm{c}}$ отвечает движению центра масс. Разлагая пространство состояний на соответству-

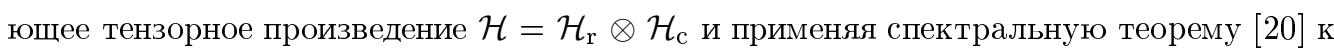
оператору $\widehat{H}_{\mathrm{r}}$ на пространстве $\mathcal{H}_{\mathrm{r}}$ и оператору $\widehat{H}_{\mathrm{c}}$ на пространстве $\mathcal{H}_{\mathrm{c}}$, мы получаем унитарно-эквивалентные данным операторы умножения на функции: $f_{\mathrm{r}}$ на пространстве $\mathcal{L}^{2}\left(M_{\mathrm{r}}, d \mu_{\mathrm{r}}\right)$ и $f_{\mathrm{c}}$ на пространстве $\mathcal{L}^{2}\left(M_{\mathrm{c}}, d \mu_{\mathrm{c}}\right)$, где $\mu_{\mathrm{r}}\left(M_{\mathrm{r}}\right), \mu_{\mathrm{c}}\left(M_{\mathrm{c}}\right)<\infty$. При этом

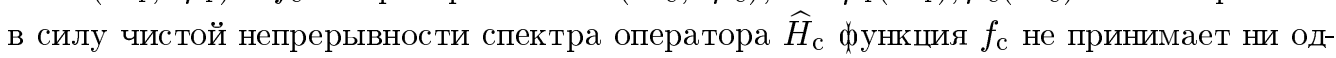
ного своего значения на множестве ненулевой меры. Таким образом, оператор $\widehat{H}$ будет унитарно-эквивалентен оператору умножения на функцию $f_{\mathrm{r}}+f_{\mathrm{c}}$ на пространстве $\mathcal{L}^{2}\left(M_{\mathrm{r}} \otimes M_{\mathrm{c}}, d \mu_{\mathrm{r}} \otimes d \mu_{\mathrm{c}}\right)$, которая также не принимает ни одного своего значения на множестве ненулевой меры. Действительно, в противном случае для некоторого числа $\sigma \in \mathbf{R}$ множество $\Sigma=\left\{(x, y) \in M_{\mathrm{r}} \times M_{\mathrm{c}} \mid f_{\mathrm{r}}(x)+f_{\mathrm{c}}(y)=\sigma\right\}$ имело бы ненулевую меру. Рассмотрим характеристическую функцию этого множества $\chi_{\Sigma}$. Из-за конечности мер множеств $M_{\mathrm{r}}$ и $M_{\mathrm{c}}$ к функции $\chi_{\Sigma}$ можно применить теорему Фубини [20] и получить

$$
\begin{aligned}
\mu_{\mathrm{r}} \otimes \mu_{\mathrm{c}}(\Sigma) & =\int_{\Sigma} \chi_{\Sigma} d \mu_{\mathrm{r}}(x) \otimes d \mu_{\mathrm{c}}(y)=\int_{M_{\mathrm{r}}}\left(\int_{M_{\mathrm{c}}} \chi_{\Sigma} d \mu_{\mathrm{c}}(y)\right) d \mu_{\mathrm{r}}(x)= \\
& =\int_{M_{\mathrm{r}}} \mu_{\mathrm{c}}\left(\Sigma_{x}\right) d \mu_{\mathrm{r}}(x)=\int_{M_{\mathrm{r}}} 0 \cdot d \mu_{\mathrm{r}}(x)=0
\end{aligned}
$$

где $\Sigma_{x}=\left\{y \in M_{\mathrm{c}} \mid f_{\mathrm{r}}(x)+f_{\mathrm{c}}(y)=\sigma\right\}$ и $\mu_{\mathrm{c}}\left(\Sigma_{x}\right)=0$. Тем самым оператор $\widehat{H}$ не имеет собственных значений. Поэтому на некомпактном пространстве $\mathbf{H}^{n}$ у двухчастичного гамильтониана с неразделяюшимися переменными естественно также ожидать отсутствия собственных значений.

\section{Список литературы}

[1] Дж. Вольф. Пространства постоянной кривизны. М.: Наука, 1982.

[2] Э. Шредингер. Избранные труды по квантовой механике. М.: Наука, 1976.

[3] L. Infeld. Phys. Rev. 1941. V. 59. P. 737.

[4] P. W. Higgs. J. Phys. A. Math. Gen. 1979. V. 12. P. 309.

[5] H. I. Leemon. J. Phys. A. Math. Gen. 1979. V. 12. P. 489

[6] J. J. Slawianowski. Bull. Acad. pol. sci. Sér. sci. phys. et astron. 1980. V. 28. № 2. P. 83.

[7] J. J. Slawianowski, J. Slominski. Bull. Acad. pol. sci. Sér. sci. phys. et astron. 1980. V. 28. № 2. P. 99.

[8] V. V. Kozlov, A. O. Harin. Celest. Mech. and Dynam. Astron. 1992. V. 54. P. 393.

[9] В. В. Козлов. Вестн. МГУ. Сер. 1. Матем. механ. 1994. № 2. С. 28.

[10] A. O. Barut, A. Inomata, G. Junker. J. Phys. A. Math. Gen. 1987. V. 20. P. 6271; 1990. V. 23. P. 1179.

[11] А. В. Щепетилов. ТМФ. 1996. Т. 109. С. 395.

[12] A. V. Shchepetilov. J. Phys. A. Math. Gen. 1998. V. 31. P. 6279.

[13] M. J. Gotay. J. Math. Phys. 1986. V. 27. № 8. P. 2051.

[14] G. M. Tuynmann. J. Math. Phys. 1989. V. 31. P. 83.

[15] Н. Я. Виленкин. Специальные функции и теория представлений групп. М.: Наука, 1991.

[16] В. Н. Говорухин, В. Г. Цибулин. Введение в Марle. Математический пакет для всех. М.: Мир, 1997. 
[17] В. В. Голубев. Лекции по аналитической теории дифференциальных уравнений. М.: ГИТТЛ, 1950.

[18] М. Рид, Б. Саймон. Методы современной математической физики. Т. 2. Гармонический анализ. Самосопряженность. М.: Мир, 1978.

[19] Л. Д. Ландау, Е. М. Лифииц. Квантовая механика. Нерелятивистская теория. М.: Наука, 1989.

[20] М. Рид, Б. Саймон. Методы современной математической физики. Т. 1. Функциональный анализ. М.: Мир, 1977.

Поступила в редакцию 5.VI.1998 г. 